\title{
Epidemiology and survival of idiopathic pulmonary fibrosis from national data in Canada
}

\author{
Robert B. Hopkins ${ }^{1,2}$, Natasha Burke ${ }^{1,2}$, Charlene Fell ${ }^{3}$, Genevieve Dion ${ }^{4}$ and \\ Martin Kolb ${ }^{5}$
}

Affiliations: ${ }^{1}$ Programs for Assessment of Technology in Health (PATH) Research Institute, St Joseph's Healthcare Hamilton, Hamilton, ON, Canada. ${ }^{2}$ Dept of Clinical Epidemiology and Biostatistics, Faculty of Health Sciences, McMaster University, Hamilton, ON, Canada. ${ }^{3}$ Division of Respirology, Dept of Medicine, University of Calgary, Calgary, AB, Canada. ${ }^{4}$ Quebec Heart and Lung Institute, Laval University, Quebec City, QC, Canada. ${ }^{5}$ Division of Respirology, Dept of Medicine, Faculty of Health Sciences, McMaster University, Hamilton, ON, Canada.

Correspondence: Robert B. Hopkins, PATH Research Institute, 25 Main St West, Suite 2000, Hamilton, ON, L8P 1H1, Canada. E-mail: hopkinramcmaster.ca

ABSTRACT Idiopathic pulmonary fibrosis (IPF) is a rare disease, with estimates of prevalence varying considerably across countries due to paucity in data collection. The aim of this study was to investigate the prevalence and incidence of IPF in Canada using administrative data requiring minimal extrapolation.

We used mandatory national administrative data from 2007-2011 to identify IPF cases of all ages with an International Classification of Diseases (Version 10, Canadian) diagnosis code of J84.1. We used a broad definition that excluded cases with subsequent diagnosis of other interstitial lung diseases, and a narrow definition that required further diagnostic testing prior to IPF diagnosis. We explored survival and quality of life.

For all ages, the broad prevalence of IPF was 41.8 per 100000 (14259 cases) and was higher for men. The incidence rate was 18.7 per 100000 (6390 cases) and was higher for men. The narrow prevalence was 20.0 per 100000 (6822 cases) and incidence was 9.0 per 100000 (3057 cases). The 4-year risk of death was $41.0 \%$ and the quality of life with IPF after 2 years was lower than for Global Initiative for Chronic Obstructive Lung Disease stage IV chronic obstructive pulmonary disease.

Using comprehensive national data, the prevalence of IPF in Canada was higher than other national estimates, suggesting that either IPF may be more common in Canada or that data capture may have been previously limited.

@ERSpublications

National data for $100 \%$ of admissions in Canada show higher prevalence and incidence of IPF than in other countries http://ow.ly/4mTVQD

Editorial comment in: Eur Respir J 2016; 48: 26-28.

This article has supplementary material available from erj.ersjournals.com

Received: Sept 092015 | Accepted after revision: April 032016 | First published online: May 262016

Support statement: The study was funded by an unrestricted research grant from InterMune Canada Inc. Funding information for this article has been deposited with FundRef.

Conflict of interest: Disclosures can be found alongside this article at erj.ersjournals.com

Copyright CERS 2016 


\section{Introduction}

Idiopathic pulmonary fibrosis (IPF) is a chronic, progressive life-threatening lung disease of unknown cause with median survival of 3-5 years from diagnosis [1], despite being the most common form of over 150 interstitial lung diseases (ILDs) [2]. For IPF to be classified as being rare, or orphan, the overall prevalence must be $<50$ per 100000 [3], but this classification remains uncertain.

With the advent of new therapies for IPF, it is crucial to obtain better data on IPF incidence and prevalence for planning of healthcare services to diagnose and treat IPF patients. Currently, there are limited and inconsistent estimates of the incidence of IPF in Europe and the USA, ranging from 0.9 to 16.3 per 100000 for all ages, depending on the case definition of IPF [4-7].

Three recent systematic reviews of the published literature indicated that most epidemiological studies of IPF are based on small sample cohort studies requiring substantial extrapolation and there is paucity of national studies to estimate the existence of IPF [8-10]. Specifically, KAUNISTO et al. [10] emphasised that most studies on IPF were made with small samples. For example, one study identified only 47 unique cases from a community registry in Minnesota (USA) that extrapolated to 90000 suspected cases in the USA. The analysis of the largest database to date for the USA by RAGHU et al. [6] was conducted on a health claims database ( $<1 \%$ of the national population), identifying 387 unique cases for a narrow definition. The largest study in the UK was a sample of primary practices from The Health Improvement Network (THIN) database, which at that time had coverage for 3 million patients [5]. The THIN study identified 2074 unique cases, which was then extrapolated to $>150000$ possible cases, a sample of $1.3 \%$ of the population. Any random sampling of cases to make a national projection would be subject to selection bias, while a national study that is comprehensive in its database coverage and that does not rely heavily on extrapolation would be helpful to set the first standard for national rates of incidence and prevalence.

A separate concern with the identification of unique cases of IPF revolves around the definition of a case. In 2000, the American Thoracic Society and the European Respiratory Society generated an international consensus statement that suggested that IPF be diagnosed with surgical lung biopsy (open thoracotomy or video-assisted thoracoscopy) or high-resolution computed tomography (CT), to exclude other ILDs [2]. This may suggest that studies conducted on databases prior to the year 2000 may be limited in relevance.

In Canada, there is mandatory standardised reporting of all hospital admissions to one common database. From this database, we can report the first comprehensive estimate of the epidemiology of IPF that involves minimal extrapolation compared with all previous reports in the literature, and provides a current robust estimate after the international consensus statement was issued. The primary objective was to estimate the prevalence and incidence for IPF in Canada in 2011. Secondary objectives were to investigate the survival and level of health-related quality of life in an incident cohort, and then make comparisons with chronic obstructive pulmonary disease (COPD).

\section{Methods}

Data sources for cases

Two national mandatory administrative databases from the Canadian Institute for Health Information (CIHI) [11] were used to identify IPF cases in Canada. Each database is built on the healthcare that was required for citizens identified by personal health card numbers. The first database, the Discharge Abstract Database (CIHI-DAD), captures $100 \%$ of acute care admissions and same day surgery in all provinces and territories across Canada, including public and private hospitals (5\% of hospitals are private) as well as the province of Quebec, which uses a different reporting database that is later translated to CIHI-DAD [12]. The second database, the National Ambulatory Care Reporting System (CIHI-NACRS), captures all emergency departments and hospital-based clinics (e.g. respirology, diagnostic) in the provinces of Alberta and Ontario [13].

\section{Cohort definition}

Both databases use International Classification of Diseases, Version 10, Canadian (ICD-10 CA) codes to identify reasons for admission and comorbidities [14]. Any patient at any age that had an ICD-10 CA J84.1 code (other interstitial pulmonary diseases with fibrosis) in any record over the 5-year period from 2007 to 2011 was captured. Subsequently, a prevalent case was identified as having healthcare with a J84.1 code in the year 2011. An incident case for the year 2011 was identified as having healthcare with a J84.1 code in the current year, without a J84.1 code in any of the previous 4 years.

A case was further defined with a broad definition, by excluding cases with an ICD-10 CA code for another ILD after the J84.1 code, similar to other studies (supplementary table S1) [6, 15]. A narrow definition further excluded cases that did not have chest CT, bronchus or lung biopsy, or bronchoscopy prior to establishing a J84.1 code [16]. 


\section{Extrapolations}

Minor extrapolations were conducted to fill in the gaps that existed because of provincial variation in data coverage. The prevalent and incident cases were established based on the national admission data and day surgery. Next, the ratio of additional cases that were identified from emergency and clinic visit data from Ontario and Alberta was estimated and then applied to the other provinces without emergency and clinic visit data. Finally, the numbers of prevalent and incident cases were estimated and reported as rates per 100000 using census data [17].

To compare our estimate of the national prevalence and incidence of IPF to previous studies with a similar case definition (IPF without other ILD), we have reported the description of the studies and rates of incidence and prevalence for comparison.

\section{Sensitivity analysis}

Sensitivity analysis was conducted for the extrapolation of incremental prevalent cases identified by emergency/clinic care that was made with an average of Ontario and Alberta rates, by exploring attributions based on Ontario alone (a lower rate) and Alberta alone (a higher rate).

\section{Mortality}

To estimate survival with Kaplan-Meier analysis, we identified an incident cohort from Ontario from 2008, which excluded any case with healthcare for IPF in 2007 and had the potential to be followed up for 4 years to the end of 2011, with death being recorded during the hospital stay or recorded as death on arrival to an emergency room.

\section{Quality of life}

For incident cases that were identified with admissions and emergency visits, we linked to the Home Care Reporting System that was available for Ontario to retrieve the patients' home care assessments over the 5-year period, 2007-2011. For all Ontario residents that receive home care visits, which included home oxygen therapy, the quarterly mandatory comprehensive assessments use the InterRai Minimum Data Set questionnaire, which captures more than 200 questions including information on quality of life domains such as mobility or pain. The data were used to estimate Health Utility Index version 2 (HUI-2) generic quality of life using an established algorithm [18], where 0 represents death and 1 represents perfect health. We reported the quality of life for the incident cohort defined for the survival analysis, and then compared quality of life with COPD patients stratified by the Global Initiative for Chronic Obstructive Lung Disease (GOLD) criteria [19].

\section{Results}

\section{Case identification}

14366 unique cases were identified from the CIHI-DAD and CIHI-NACRS databases for the broad case definition cohort for the year 2011 (figure 1a). 1710 cases had a different ILD diagnosis subsequent to recording the J84.1 code and were excluded (table S1). After extrapolation of additional cases for provinces without records for emergency room visits and hospital-based clinics, an additional 1603 cases were extrapolated for a total of 14259 cases (table S2). Among the IPF cases that met the broad definition $(n=14259), 6822$ narrow definition cases included a record of at least one diagnostic procedure $(40 \%$ had undergone CT, 35.2\% had biopsy, 57.3\% had either CT or biopsy and 11.5\% had bronchoscopy).

For the incident cohort, 5781 unique cases were identified in 2011; however, 510 were excluded because of a subsequent diagnosis of other ILD (figure 1b) and 1119 were added due to extrapolation for provinces without records for emergency room visits and hospital-based clinics (table S3). The national estimate of incidence using the broad definition was 6390 cases, of which 3057 were included as being narrow definition with a recorded diagnostic test before IPF diagnosis.

\section{Extrapolations}

In Ontario and Alberta, which had the most complete datasets, the prevalent cohort had a mean of 1.3 acute care admissions and 7.5 visits to emergency/clinics in the year 2011. The incident cohort had on average 1.5 acute care admissions and 7.9 visits to emergency/clinics in their index year. Thus, more records were available from the emergency/clinics setting to have the possibility of a J84.1 code than existed in the acute admission records. In Ontario and Alberta, 81\% of all prevalent cases reported an acute care admission or day surgery with a J84.1 code, and there was an incremental $20 \%$ cases identified by emergency/clinics. 
a)

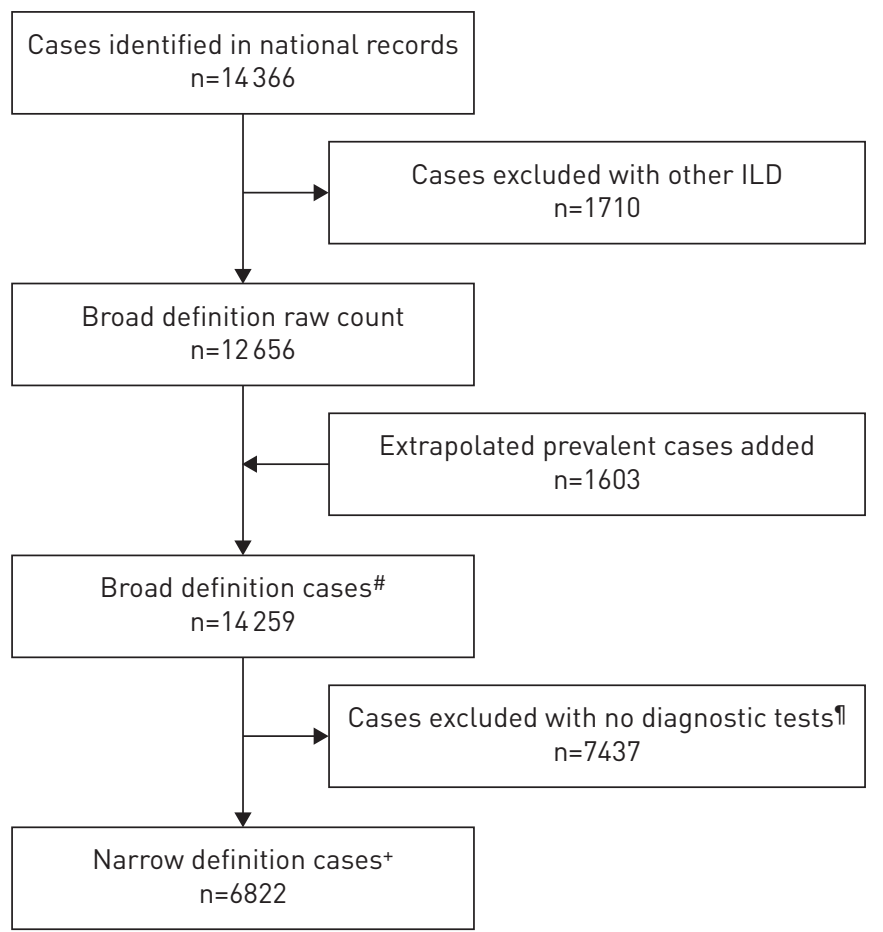

b)

Cases identified in national records
\[ \mathrm{n}=5781 \]

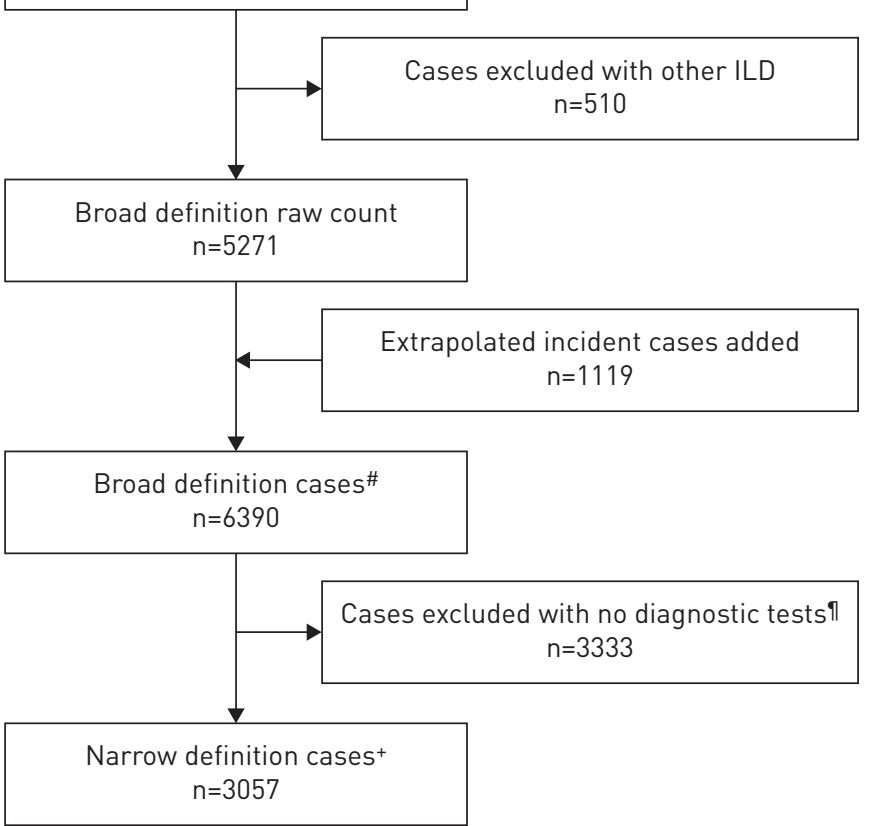

FIGURE 1 Case identification for idiopathic pulmonary fibrosis (IPF) a) prevalence and b) incidence. ILD: interstitial lung disease. \#: any diagnosis code of IPF, not followed by diagnosis code for other interstitial disease; ${ }^{\text {I: }}$ cases where no computed tomography (CT), biopsy or bronchoscopy were recorded in any admission record at any time for each case; ${ }^{+}$: broad definition plus IPF diagnosis listed after CT, bronchoscopy or biopsy.

\section{Prevalent and incident cases}

The number of prevalent cases in 2011 based on a broad IPF definition was 6571 women (38.2 per 100000 all ages) and 7688 men (45.4 per 100000) for a total of 14259 (41.8 per 100000) (table 1). Following the same methodology used for prevalent cases, the number of incident IPF cases based on a broad definition in 2011 was 2791 women (16.2 per 100000) and 3599 men (21.3 per 100000) for a total of 6390 (18.7 per 100000).

The number of prevalent cases in 2011 based on a narrow IPF definition was 3047 women (17.7 per 100000 all ages) and 3775 men (22.3 per 100000) for a total of 6822 (20.0 per 100000) (table 1). Following the same methodology used for prevalent cases, the number of incident IPF cases based on a narrow definition in 2011 was 1273 women (7.4 per 100000) and 1784 men (10.5 per 100000) for a total of 3057 (9.0 per 100 000).

For both men and women the prevalence rose with age, with a mean \pm sD age at first record of $75.0 \pm 13.8$ years for women and $74.0 \pm 12.8$ years for men. The rate of prevalence for both men and women, using a broad IPF definition, increased with age from 25.9 per 100000 population for age 50-59 years for both men and women to 507.0 per 100000 population at age $\geqslant 90$ years (table 1 ). Similar patterns existed for prevalent cases with a narrow definition. Conversely, the rate of incidence for narrowly defined cases was relatively constant across age groups for women but the rate of incidence accelerated by age for men.

The rates of prevalence for cases with narrow definition varied across provinces, with lower rates estimated in the western provinces of British Columbia, Alberta, Saskatchewan and Manitoba (ranging 31-40 per 100000 at age $\geqslant 50$ years for men, and $27-35$ per 100000 at age $\geqslant 50$ years for women) (figure 2). The highest rates were seen in Quebec, where the prevalence was 88 per 100000 men aged $\geqslant 50$ years and 64 per 100000 women aged $\geqslant 50$ years.

\section{Sensitivity analysis of prevalence and incidence estimates}

For the primary estimate of incidence and prevalence, we extrapolated the incremental cases that were identified by emergency room care from the provinces that reported (Ontario and Alberta). The extrapolation was based on the average incremental percentages across the two provinces. As sensitivity analysis, when we used only Ontario or only Alberta, the difference was only 56 cases, or $<0.4 \%$ difference. 
TABLE 1 National estimates of prevalence and incidence of idiopathic pulmonary fibrosis for Canada, 2011

Age group years

\begin{tabular}{lc}
\hline & Broad $^{\#}$ \\
\hline Cases & Rate \\
& per 100000
\end{tabular}

Prevalence

\begin{tabular}{cc} 
Narrow $^{\text {I }}$ \\
\hline Cases & $\begin{array}{c}\text { Rate } \\
\text { per } 100000\end{array}$
\end{tabular}

Incidence

\begin{tabular}{|c|c|c|c|}
\hline \multicolumn{2}{|c|}{ Broad $^{\#}$} & \multicolumn{2}{|c|}{ Narrow } \\
\hline Cases & $\begin{array}{c}\text { Rate } \\
\text { per } 100000\end{array}$ & Cases & $\begin{array}{c}\text { Rate } \\
\text { per } 100000\end{array}$ \\
\hline
\end{tabular}

\begin{tabular}{|c|c|c|c|c|c|c|c|c|}
\hline \multicolumn{9}{|l|}{ Women } \\
\hline $0-19$ & 33 & 0.9 & 23 & 0.6 & 17 & 0.4 & 9 & 0.2 \\
\hline $20-29$ & 47 & 2.0 & 25 & 1.1 & 32 & 1.4 & 21 & 0.9 \\
\hline $30-39$ & 85 & 3.7 & 53 & 2.3 & 53 & 2.3 & 35 & 1.5 \\
\hline $50-59$ & 574 & 23.2 & 375 & 15.2 & 309 & 12.5 & 184 & 7.5 \\
\hline $60-69$ & 1095 & 62.2 & 631 & 35.9 & 576 & 32.7 & 316 & 18.0 \\
\hline $70-79$ & 1747 & 161.0 & 839 & 77.4 & 792 & 73.0 & 363 & 33.5 \\
\hline Total & 6571 & 38.2 & 3047 & 17.7 & 2791 & 16.2 & 1273 & 7.4 \\
\hline \multicolumn{9}{|l|}{ Men } \\
\hline $0-19$ & 58 & 1.4 & 25 & 0.6 & 34 & 0.8 & 15 & 0.4 \\
\hline $20-29$ & 40 & 1.6 & 28 & 1.2 & 22 & 0.9 & 12 & 0.5 \\
\hline $30-39$ & 78 & 3.4 & 44 & 1.9 & 50 & 2.2 & 27 & 1.2 \\
\hline $40-49$ & 196 & 7.5 & 123 & 4.7 & 129 & 4.9 & 78 & 3.0 \\
\hline$\geqslant 90$ & 453 & 763.0 & 156 & 262.7 & 204 & 343.6 & 103 & 173.6 \\
\hline Total & 7688 & 45.4 & 3775 & 22.3 & 3599 & 21.3 & 1784 & 10.5 \\
\hline \multicolumn{9}{|c|}{ Women and men } \\
\hline $0-19$ & 91 & 1.2 & 48 & 0.6 & 51 & 0.7 & 24 & 0.3 \\
\hline $20-29$ & 87 & 1.8 & 53 & 1.1 & 54 & 1.1 & 33 & 0.7 \\
\hline $30-39$ & 163 & 3.5 & 97 & 2.1 & 103 & 2.2 & 62 & 1.4 \\
\hline $40-49$ & 447 & 8.6 & 295 & 5.7 & 268 & 5.2 & 176 & 3.4 \\
\hline $50-59$ & 1271 & 25.9 & 796 & 16.2 & 699 & 14.3 & 423 & 8.6 \\
\hline $60-69$ & 2602 & 75.7 & 1552 & 45.2 & 1355 & 39.5 & 756 & 22.0 \\
\hline $70-79$ & 4030 & 199.8 & 1923 & 95.4 & 1989 & 98.7 & 928 & 46.1 \\
\hline $80-89$ & 4462 & 400.0 & 1727 & 154.9 & 1521 & 136.4 & 520 & 46.6 \\
\hline$\geqslant 90$ & 1106 & 507.0 & 326 & 149.5 & 341 & 156.4 & 133 & 61.0 \\
\hline Total & 14259 & 41.8 & 6822 & 20.0 & 6390 & 18.7 & 3057 & 9.0 \\
\hline
\end{tabular}

Mortality

In the incident cohort of Ontario for 2008, where data was most complete, based on the narrow definition cases that were identified in the second year and followed for up to 4 years, $21 \%$ of patients died in hospital during the first year (18.8\% women, $24.3 \%$ men) (figure 3 and table 2$)$. By the end of the fourth year, $41 \%$ had died in hospital ( $45.5 \%$ men, $39.8 \%$ women).

At the national level in the year 2011, the total number of deaths for patients with IPF was 3051, which represents $1.4 \%$ of all deaths in Canada. In comparison, in 2007, which was the most recent year with available national data, the number of deaths that were due to COPD (ICD-10 CA J44 to J47) for those aged $\geqslant 50$ years was 10546 ( $4.8 \%$ of all deaths in Canada), with COPD being the fourth leading cause of death [20]. The total national deaths for patients with IPF was $28.9 \%$ of the total national number of deaths from COPD, while the rate of prevalence of IPF was only $2.9 \%$ of the rate of prevalence of COPD (0.11\% IPF versus $4 \%$ COPD), underscoring the extremely high mortality associated with an IPF diagnosis.

\section{Quality of life}

The health-related quality of life for women and men combined as measured using the HUI-2 algorithm for the Ontario incident cohort was 0.585 in the first year and declined to 0.432 by the fourth year, being lower for women than men (figure 4 and table 3). This baseline HUI-2 reflects the lower quality of life than national average from the Canadian Community Health Survey for a similar distribution by age for 


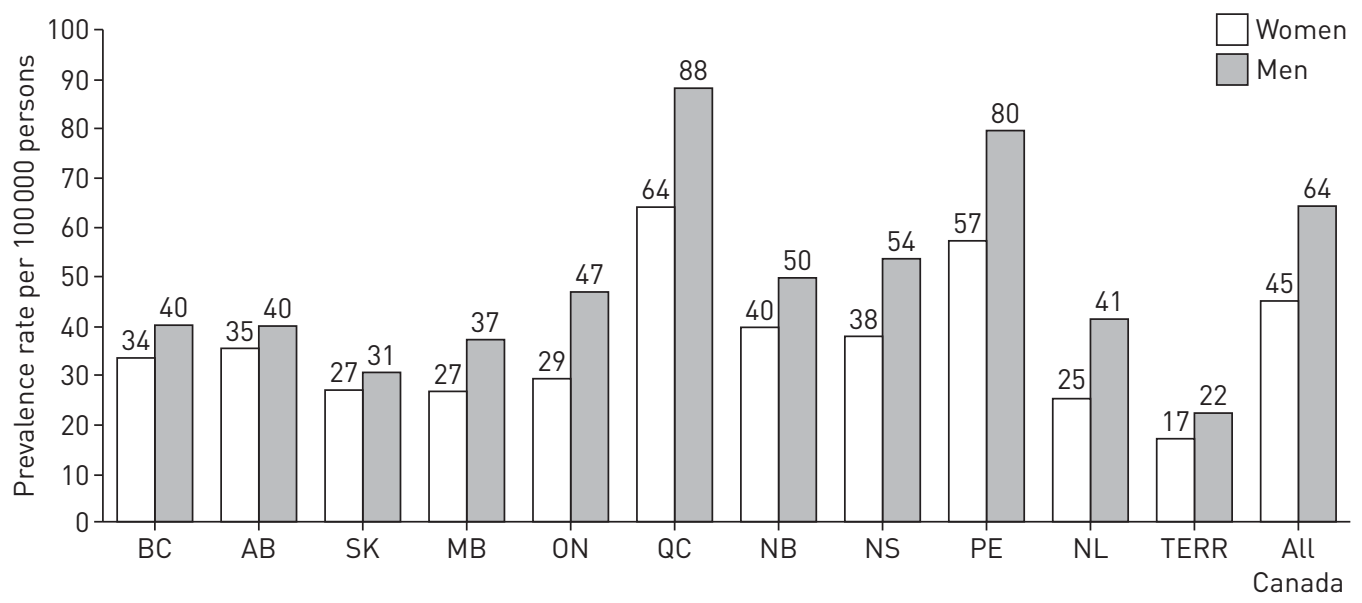

FIGURE 2 Prevalence rates of idiopathic pulmonary fibrosis by the narrow definition, per 100000 persons aged $\geqslant 50$ years, by province and sex, in 2011. BC: British Columbia; AB: Alberta; SK: Saskatchewan; MB: Manitoba; ON: Ontario; QC: Quebec; NB: New Brunswick; NS: Nova Scotia; PE: Prince Edward Island; NL: Newfoundland and Labrador; TERR: territories.

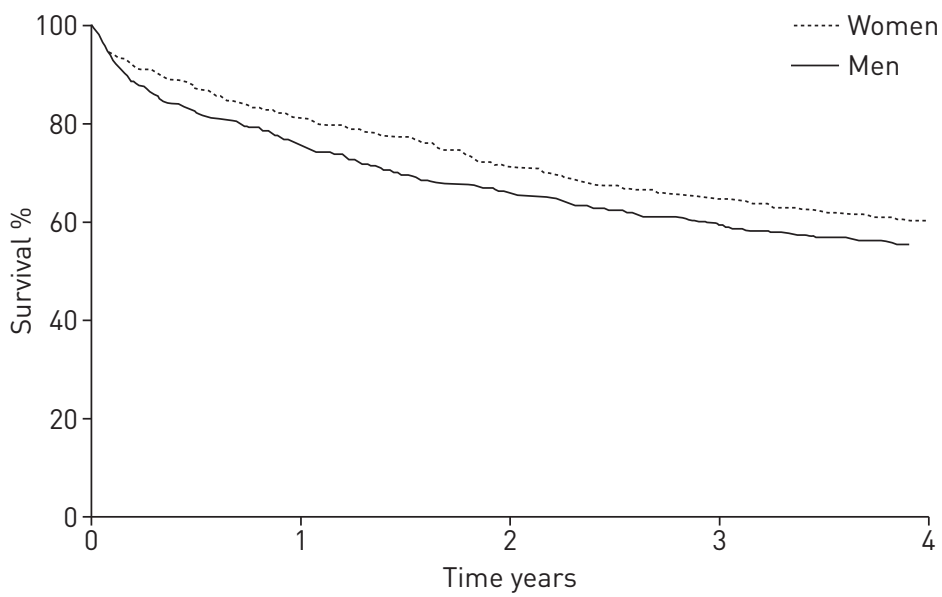

FIGURE 3 Kaplan-Meier survival estimates for incident idiopathic pulmonary fibrosis (IPF) cases identified in 2008 in the province of Ontario, using a narrow case definition, aged $\geqslant 50$ years. The incident cohort was defined for 2008 with 2007 as the pre-IPF exclusion period for incidence. Cases were followed for a maximum of 4 years, from the start of 2008 to the end of 2011. For data details, see table 2.

TABLE 2 Kaplan-Meier survival estimates

Time years

\begin{tabular}{ccccc}
\hline $\mathbf{0}$ & $\mathbf{1}$ & $\mathbf{2}$ & $\mathbf{3}$ & $\mathbf{4}$ \\
\hline 613 & & & & \\
0 & 464 & 404 & 366 & 340 \\
$0 \%$ & 149 & 209 & 247 & 273 \\
$100.0 \%$ & $24.3 \%$ & $34.1 \%$ & $40.3 \%$ & $45.5 \%$ \\
538 & $75.7 \%$ & $65.9 \%$ & $59.7 \%$ & $55.5 \%$ \\
0 & & & & \\
$0 \%$ & 437 & 383 & 348 & 324 \\
$100.0 \%$ & 101 & 155 & 190 & 214 \\
1151 & $18.8 \%$ & $28.8 \%$ & $35.3 \%$ & $39.8 \%$ \\
0 & $81.2 \%$ & $71.2 \%$ & $64.7 \%$ & $60.2 \%$ \\
$0 \%$ & 901 & 787 & 714 & 664 \\
$100.0 \%$ & 250 & 364 & 437 & 487 \\
& $21.0 \%$ & $30.5 \%$ & $36.8 \%$ & $41.0 \%$ \\
& $79.0 \%$ & $69.5 \%$ & $63.2 \%$ & $59.0 \%$ \\
\hline
\end{tabular}




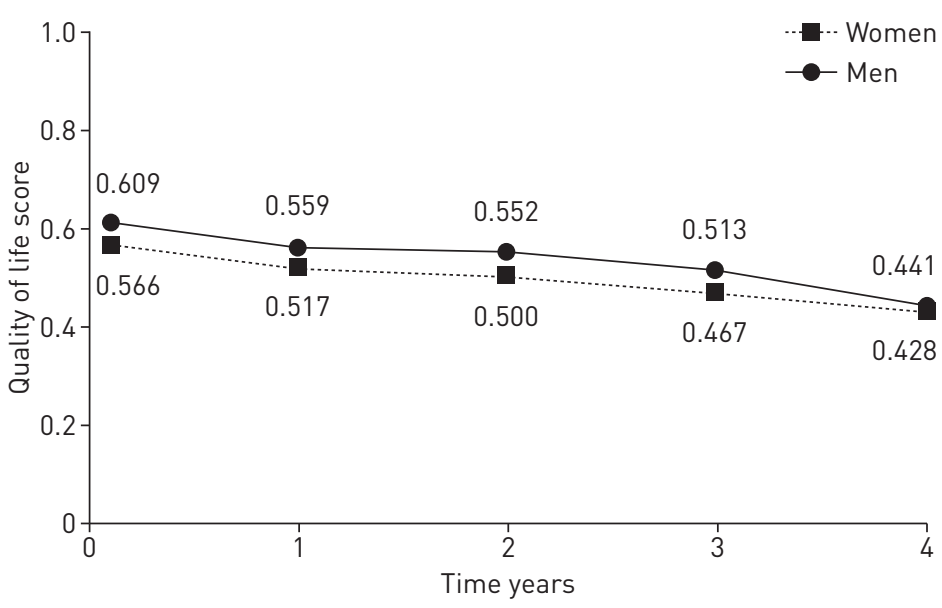

FIGURE 4 Health-related quality of life scores according to the Health Utility Index version 2, for the incident cohort of idiopathic pulmonary fibrosis (IPF) patients in the province of Ontario. The incident cohort was defined for 2008 with 2007 as the pre-IPF exclusion period for incidence. Cases were followed for a maximum of 4 years, from the start of 2008 to the end of 2011. Reference quality of life scores using the Global Initiative for Chronic Obstructive Lung Disease criteria for chronic obstructive pulmonary disease are: stage I (forced expiratory volume in $1 \mathrm{~s}$ (FEV 1 ) $>80 \%$ predicted) 0.84 ; stage II (FEV $150-79 \%$ pred) 0.73 ; stage III (FEV $30-49 \%$ pred) 0.74 ; stage IV (FEV1 $<30 \%$ pred) 0.52 . For data details, see table 3 .

\begin{tabular}{|c|c|c|c|c|c|}
\hline & \multicolumn{5}{|c|}{ Time years } \\
\hline & 0 & 1 & 2 & 3 & 4 \\
\hline \multicolumn{6}{|l|}{ Women } \\
\hline Patients & 2030 & 1183 & 937 & 505 & 190 \\
\hline Score & $0.566 \pm 0.005$ & $0.517 \pm 0.006$ & $0.500 \pm 0.006$ & $0.467 \pm 0.009$ & $0.428 \pm 0.015$ \\
\hline \multicolumn{6}{|l|}{ Men } \\
\hline Patients & 1712 & 840 & 552 & 263 & 83 \\
\hline Score & $0.609 \pm 0.005$ & $0.559 \pm 0.007$ & $0.552 \pm 0.008$ & $0.513 \pm 0.012$ & $0.441 \pm 0.020$ \\
\hline
\end{tabular}

Data are presented as $\mathrm{n}$ or mean $\pm \mathrm{SE}$.

all Canadians of 0.741 , but is similar to the average for all residents who require supportive care of 0.597. In comparison, the overall quality of life for COPD patients with GOLD stage III (forced expiratory volume in $1 \mathrm{~s}$ of $30-49 \%$ predicted) was 0.74 and higher for stages I and II. This suggests that the incident cohort of IPF patients have quality of life in their first year of diagnosis lower than GOLD stage III COPD. Beyond year 2, the quality of life for IPF patients was substantially below the average for GOLD stage IV COPD patients (0.52), and continued to decline.

Rates of prevalence and incidence from previous studies are presented in table S4. In comparison to the previous estimates, we see that they all were lower estimates of prevalence and incidence of IPF than we estimate for Canada. If we take the study with the largest rate of prevalence, by RAGHU et al. [6], with a rate of prevalence with a broad definition of 42.7 per 100000 at age $\geqslant 18$ years, this predicts 11183 cases, or $78 \%$ of the Canadian estimate of 14259 cases. All of the other studies except RaGHu et al. [6] would predict $<50 \%$ of the actual total.

\section{Discussion}

Based on our analysis of the data from the mandatory national reporting of institutional acute care in Canada, we estimated that there were 14259 prevalent cases and 6390 incident cases of IPF in the year 2011. This corresponds to a rate per 100000 population for all ages of 41.8 for prevalence and 18.7 for incident cases. The rate of prevalence was about $19 \%$ higher in men (45.4 cases per 100000) than women (38.2 per 100000$)$. The rate of incidence was $31 \%$ higher in men $(21.3$ per 100000$)$ than women $(16.2$ per $100000)$. We report that there are few cases aged $<50$ years, and rare cases aged $<19$ years of age may have a genetic origin, despite using similar coding to establish cases.

Previous research has reported the rates of prevalence and incidence in IPF in other jurisdictions using different methods (table S4). A recent literature review pointed out that many of the estimates relied on 
large extrapolations for the prevalence of IPF [9]. For example, RAGHU et al. [6] relied on a health claims database that covered $<1 \%$ of the population of the USA, while studies conducted based on the UK THIN databases covered 255 general practices, or $3 \%$ of the population [21]. Currently, the THIN data contain the electronic medical records of 11.1 million patients collected from 562 general practices in the UK, covering $6.2 \%$ of the UK population [22]. If we had relied on previous studies with less data capture, our estimates may have been underreported by $50 \%$ or more.

However, there is provincial variation, which suggests that there may also be country level variation in the estimates of IPF prevalence and incidence, if studies similar to our study are conducted in the future elsewhere. The highest rates were seen in the province of Quebec (figure 2), which, along with Ontario, is a major manufacturing industrial province in Canada (populations 13 million and 8 million, respectively). Since the general health of people in the provinces is similar, with life expectancy differences being $<2$ years between all provinces, variation in the prevalence may be due to differences in the delivery of healthcare services, with the smaller eastern maritime provinces of New Brunswick ( 0.7 million), Nova Scotia (0.9 million), Prince Edward Island ( 0.1 million), and Newfoundland and Labrador ( 0.5 million) perhaps having closer access to hospital-based care instead of dispersed community-based care, creating greater data capture of cases.

There are strengths and limitations in the analysis when using national administrative institutional databases. In our study, we were able to capture $100 \%$ of all admissions and day surgeries (including visits for advanced diagnostic testing in hospital). Data coverage was not comprehensive for emergency room visits across all provinces, with only Ontario and Alberta having mandatory reporting. Overall, the additional cases that were identified by extrapolation for missing emergency room visits added $13 \%$ for broad prevalence $(n=1603)$ and $21 \%$ for broad incidence $(n=1119)$ (figure 1). However, the percentage increases in the number of extra cases that were identified using either Ontario or Alberta data for emergency visits were similar, which strengthened the method of extrapolation of extra cases across Canada based on emergency room visits from these two provinces. Still, since there is provincial variation in healthcare systems, there may be some variability not identifiable in the databases.

The identification of cases was limited by coding of admissions and lack of linkage to physician records. In our analysis, we had access to available ICD-10 CA codes, which may be more specific than ICD-9 codes used in other work in the USA [6]. In particular, the term IPF was not included in the ICD-9 codes, and ICD-9 codes may classify more patients with other common ILDs such as diffuse pulmonary fibrosis. However, the ICD-10 CA code J84.1 is defined as "other interstitial pulmonary diseases with fibrosis", which includes "diffuse pulmonary fibrosis", "fibrosing alveolitis (cryptogenic)", "Hamman-Rich syndrome", "IPF" and "usual interstitial pneumonia", with an unknown percentage of diseases other than IPF.

In addition, unlike other work, we did not have access to physician billing records, which are only available in provincial databases. Future research on linking institutional care with physician billings at the provincial level is ongoing, and could produce higher estimates of prevalence and incidence for cases undergoing physician care and not yet requiring acute institutional care, or under physician care without a recent admission. We can speculate on the extra possible cases based on work by COLLARD et al. [15], where $52.9 \%$ of prevalent cases of IPF had an admission and $37.0 \%$ had emergency care, leaving the residual cases $(10.1 \%)$ identified by physician care alone. We are unaware of any epidemiological study that stratifies the case definition by source of care.

Using diagnostic tests to refine the case definition may underestimate the rates of IPF, as the recording of diagnostic tests is not mandatory for admissions, as we have demonstrated for other diseases. For example, in our previous work using national admissions data, we found that there was $<1 \%$ reported use of radiography for hip fracture, while admissions for hip fracture that require surgical repair often require three radiographs (pre-surgery, post-surgery and follow-up) [23], i.e. diagnostic tests that are common for each type of admission are not always separately reported. Conversely, the administrative databases do not provide test results and the use of CT, biopsy or bronchoscopy may not confirm a J84.1 code and we may have included IPF where the test results may have been negative, which suggests an overestimate. When compared with previous studies, the ratio of narrowly defined cases to broadly defined cases was higher in our study: $48 \%$ for prevalence and $48 \%$ for incidence. Other studies report $33 \%$ for prevalence and $42 \%$ for incidence $[6,15]$. The key difference may be the level of care that was being provided in our study, with all patients being identified as having any one of an acute care admission, day surgery, emergency room or in-hospital clinic visit. Within these settings, more diagnostic tests may have been ordered. The extent of resource utilisation and economic impact of IPF in Canada warrants further study.

One key finding in our analysis was the high rate of death following the diagnosis of IPF. In 2011, 3051 persons aged $\geqslant 50$ years died with IPF. For comparison, 10546 persons aged $\geqslant 50$ years died of COPD, the fourth leading cause of death, in 2007 [20]. While the rate of death is high for IPF in this analysis, we may have underestimated the risk of death because we only captured hospital/emergency room deaths and may have 
excluded deaths that occur at home. In contrast, the rate of prevalence of IPF was only $2.9 \%$ of the rate of prevalence of COPD. Despite the lower prevalence, the survival from IPF was poor ( $41 \%$ death at 4 years), confirming that the diagnosis of IPF would be devastating and that a delayed diagnosis could be too late to receive any remedial care. Furthermore, the quality of life of IPF patients is already poor and worsens over time.

\section{Conclusion}

We have used a robust approach for data collection that is uniquely available because of the universal national healthcare system in Canada. From our standardised mandatory reporting for $100 \%$ of all admissions to all hospitals, we report that the prevalence and incidence of IPF in Canada were higher than reported elsewhere in similar countries. Finally, we highlight that the impact on the patient who receives a diagnosis of IPF, in terms of expected poor survival and low quality of life, would be disastrous.

\section{Acknowledgements}

National administrative data were provided by the Canadian Institute for Health Information.

Author contributions were as follows. Conception and design: R.B. Hopkins, M. Kolb and N. Burke. Analysis and interpretation: R.B. Hopkins, M. Kolb, C. Fell and G. Dion. Drafting the manuscript for important intellectual content: R.B. Hopkins, N. Burke, C. Fell, M. Kolb and G. Dion. Final approval: R.B. Hopkins, N. Burke, C. Fell, M. Kolb and G. Dion.

\section{References}

1 Ley B, Collard HR, King TE Jr. Clinical course and prediction of survival in idiopathic pulmonary fibrosis. Am J Respir Crit Care Med 2011; 183: 431-440.

2 Raghu G, Collard HR, Egan JJ, et al. An official ATS/ERS/JRS/ALAT statement: idiopathic pulmonary fibrosis: evidence-based guidelines for diagnosis and management. Am J Respir Crit Care Med 2011; 183: 788-824.

3 107th United States Congress. Public Law 107-280: Rare Diseases Act of 2002 (House Report 107-543). Washington, United States Government Printing Office, November 6, 2002. Available from www.govtrack.us/ congress/bills/107/hr4013

4 Fernández Pérez ER, Daniels CE, Schroeder DR, et al. Incidence, prevalence, and clinical course of idiopathic pulmonary fibrosis: a population-based study. Chest 2010; 137: 129-137.

5 Navaratnam V, Fleming KM, West J, et al. The rising incidence of idiopathic pulmonary fibrosis in the UK. Thorax 2011; 66: 462-467.

6 Raghu G, Weycker D, Edelsberg J, et al. Incidence and prevalence of idiopathic pulmonary fibrosis. Am J Respir Crit Care Med 2006; 174: 810-816.

7 von Plessen C, Grinde O, Gulsvik A. Incidence and prevalence of cryptogenic fibrosing alveolitis in a Norwegian community. Respir Med 2003; 97: 428-435.

8 Olson AL, Swigris JJ. Idiopathic pulmonary fibrosis: diagnosis and epidemiology. Clin Chest Med 2012; 33: 41-50.

9 Nalysnyk L, Cid-Ruzafa J, Rotella P, et al. Incidence and prevalence of idiopathic pulmonary fibrosis: review of the literature. Eur Respir Rev 2012; 21: 355-361.

10 Kaunisto J, Salomaa ER, Hodgson U, et al. Idiopathic pulmonary fibrosis - a systematic review on methodology for the collection of epidemiological data. BMC Pulm Med 2013; 13: 53.

11 Canadian Institute for Health Information (CIHI). CIHI Annual Report, 2011-2012. At the Heart of Data. Ottawa, CIHI, 2012. https://secure.cihi.ca/free_products/AnnualReport2012-ENweb.pdf

12 Canadian Institute for Health Information (CIHI). Data Quality Documentation for External Users: Discharge Abstract Database, 2010-2011. Ottawa, CIHI, 2011. www.cihi.ca/en/dad_executive_sum_10_11_en.pdf

13 Canadian Institute for Health Information (CIHI). Data Quality Documentation for External Users: National Ambulatory Care Reporting System, 2010-2011. Ottawa, CIHI, 2011. www.cihi.ca/en/nacrs_exec_summ_2010_ 2011_en.pdf

14 Canadian Institute for Health Information (CIHI). ICD-10-CA. International Statistical Classification of Diseases and Related Health Problems, Tenth Revision, Canada. Volume Two - Alphabetical Index. Ottawa, CIHI, 2012. www.cihi.ca/en/icd_volume_two_2012_en.pdf

15 Collard HR, Ward AJ, Lanes S, et al. Burden of illness in idiopathic pulmonary fibrosis. J Med Econ 2012; 15: 829-835.

16 Canadian Institute for Health Information (CIHI). CCI. Canadian Classification of Health Interventions. Volume Four - Alphabetical Index. Ottawa, CIHI, 2012. www.cihi.ca/en/cci_volume_four_2012_en.pdf

17 Statistics Canada. Table 051-0001: Estimates of Population, by Age Group and Sex for July 1, Canada, Provinces and Territories. Annual (persons unless otherwise noted). CANSIM (database). Date last updated: September 29, 2015. Date last accessed: March 30, 2016. Available from www5.statcan.gc.ca/cansim/a26?lang=eng\&id=510001

18 Horsman J, Furlong W, Feeny D, et al. The Health Utilities Index (HUI): concepts, measurement properties and applications. Health Qual Life Outcomes 2003; 1: 54.

19 Ståhl E, Lindberg A, Jansson SA, et al. Health-related quality of life is related to COPD disease severity. Health Qual Life Outcomes 2005; 3: 56.

20 Statistics Canada. Table 102-0561: Leading Causes of Death, Total Population, by Age Group and Sex, Canada. Annual. CANSIM (database). Date last updated: December 10, 2015. Date last accessed: March 30, 2016. Available from www5.statcan.gc.ca/cansim/a26?lang=eng\&id=1020561

21 Gribbin J, Hubbard RB, Le Jeune I, et al. Incidence and mortality of idiopathic pulmonary fibrosis and sarcoidosis in the UK. Thorax 2006; 61: 980-985.

22 The Health Improvement Network (THIN) Research Team. THIN Database. www.ucl.ac.uk/pcph/research-groupsthemes/thin-pub/database Date last updated: April 14, 2015. Date last accessed: March 30, 2016.

23 Tarride JE, Hopkins RB, Leslie WD, et al. The burden of illness of osteoporosis in Canada. Osteoporos Int 2012; 23: 2591-2600. 